\title{
Introduction to the thematic issue
}

\author{
Home-based health and wellness measurement and monitoring
}

John O'Donoghue ${ }^{\mathrm{a},{ }^{*}}$, Reiner Wichert ${ }^{\mathrm{b}}$ and Monica Divitini ${ }^{\mathrm{c}}$

${ }^{a}$ Health Information Systems Research Centre, University College Cork, Ireland

${ }^{\mathrm{b}}$ Fraunhofer-Institut für Graphische Datenverarbeitung IGD, Fraunhoferstr. 5, 64283 Darmstadt, Germany

${ }^{\mathrm{c}}$ Department of Computer and Information Science, NTNU, Sem Salandsvei 7-9, NO-7491 Trondheim, Norway

\section{Introduction}

Traditional healthcare environments are extremely complex and challenging to manage, as they are required to cope with an assortment of patient conditions under various circumstances with a wide range of resource constraints. Pervasive healthcare technologies seek to respond to a variety of these pressures by successfully integrating them within existing health care environments.

This thematic issue focuses on the deployment of technologies at home which can have a positive impact on the health of our population, with a strong focus on technological developments aiming at increasing the health and wellbeing of those living at home from a variety of perspectives, for example, the collection and processing of ambient and spatial-activity datasets thought the usage of Body Area Network devices, the development and assessment of handheld mobile devices as part of the dementia screening process and finally the correlation and assessment of larger datasets through the development of data mining and simulation techniques.

Ambient Assisted Living "is currently one of the important research and development areas, where accessibility, usability and learning play a major role and where future interfaces are an important concern for applied engineering. The general goal of ambient assisted living solutions is to apply ambient intelligence technology to enable people with specific demands, e.g. handicapped or elderly, to live in their preferred environment longer" [1].

\footnotetext{
*Corresponding author. E-mail: john.odonoghue@ucc.ie.
}

\section{In this thematic issue}

Within this thematic issue five novel papers are presented. The first two papers build on the growing synergies of ambient intelligence (AmI) and Wireless Sensor technologies and AAL. As the deployment of wireless sensing devices begins to increase they are now having an impact on our way of living across many healthcare applications. To help maximise the value of these rich datasets, Wireless Sensors Networks (WSN), pervasive computing, and artificial intelligence as individual research domains have come together to build an interdisciplinary concept of AmI [2].

Authors Kaluza and Gams presented their findings on the "Analysis of Daily-Living Dynamics" which assesses the wellbeing of an elderly person that lives at home alone. Their paper presents an approach to monitoring an individual in the home environment by an AmI system in order to detect anomalies in daily-living patterns. The proposed method is based on transforming the sequence of posture and spatial information using a novel matrix presentation to extract spatial-activity features. Their experiments indicate that the proposed algorithm successfully discriminates between the daily behaviour patterns of a healthy person and those with health problems.

The second paper authored by Pogorelc and Gams, titled "Home-Based Health Monitoring of the Elder-ly through Gait Recognition" demonstrates that alternative, elderly care can be provided through home-based, automatic, health-monitoring systems. Through the usage of data-mining algorithms the automatic recognition of health problems, activities and falls through the analysis of gait can be achieved.

For a number of years the "potential benefit of mobile and wireless information technology for health- 
care service delivery, improving patient safety and reducing cost is increasingly recognised and emphasised" [3]. The third paper, authored by Kim, Hsiao and Do and titled "Home-based Computerized Cognitive Assessment Tool for Dementia Screening", clearly demonstrates the advantages which can be made by using such technologies. Up until now the 'clock drawing test', a paper-and-pencil test, has been used as one of the most popular cognitive screening tools for dementia. Presented in this paper is a home-based computerised dementia screening tool (i.e. the ClockMe System), which was developed based on an observational study of the current practice of dementia screening at a clinic.

The final two papers present two novel techniques in the assessment and processing of larger datasets to assist end users within an AmI environment. Paper four authored by Chu, Song, Levinson and Kautz titled "Interactive Activity Recognition and Prompting to Assist People with Cognitive Disabilities" presents a model of interactive activity recognition and prompting for use in an assistive system for persons with cognitive disabilities. The system is capable of determining the user's state by interpreting sensor data and/or by explicitly querying the user, and can prompt the user to begin, resume, or end tasks. The results show that the system is able to successfully guide a person with cognitive disabilities through sample schedules by delivering appropriate prompts while efficiently dealing with ambiguous situations.

Finally paper five, authored by ter Horst and Sinitsyn titled "Structuring reasoning for interpretation of sensor data in home-based health and well-being monitoring applications" presents an approach to structuring knowledge and reasoning for high-level interpretation of sensor data in e.g. independent living applications. The main contribution is to use generalised events, described in terms of 'space-time chunks', as a unifyingand simplifying structuring principle. Two applications were built and evaluated using the approach described in the paper, both of which are related to monitoring well-being of elderly people, and both of which use simple, low-cost sensors.

\section{Acknowledgments}

The guest editors wish to thank the many colleagues who have taken their precious time to review the papers for this thematic issue. The thematic issue of the
Journal of Ambient Intelligence and Smart Environments would not have been possible without the help and determination of the editorial staff and last but not the least, we would like also to thank all the authors for their hard and highly valuable work.

The guest editors wish to thank the many colleagues who have taken their precious time to review the papers for this thematic issue.

\section{Giacomo Cabri}

University of Modena and Reggio Emilia, Italy

Lorenzo Chiari

University of Bologna, Italy

Luis Castro

Computer Science Department, Ensenada, Mexico

Lynne Coventry

Northumbria University, UK

Babak Farshchian

SINTEF ICT, Trondheim, Norway

Rune Fensli

University of Agder, Norway

Francesco Furfari

ISTI CNR, Italy

Mark Hawley

University of Sheffield, UK

Cristian Hofmann

Fraunhofer-Institut IGD, Germany

Felix Kamieth

Fraunhofer-Institut IGD, Germany

Michael John

Fraunhofer FIRST, Germany

Mario Kolberg

University of Stirling, Scotland, UK

Chengzhi Liu

Norwegian University of Science and Technology

Norway

Paul J. McCullagh

University of Ulster, UK

Simone Mora

Norwegian University of Science and Technology

Norway

Michael O' Grady

University College Dublin, Ireland 
Elizabeth Perkins

University of Liverpool, UK

Emanuel Popovici

University College Cork, Ireland

Madhu Reddy

The Pennsylvania State University, USA

Andreas Schmidt

Karlsruhe University of Applied Sciences, Germany

Eric Wade

University of Southern California, USA

\section{References}

[1] T. Kleinberger, M. Becker, E. Ras, A. Holzinger, and P. Müller, Ambient intelligence in assisted living: Enable elderly people to handle future interfaces, in: Universal Access in HumanComputer Interaction. Ambient Interaction, Lecture Notes in Computer Science, Vol. 4555, Springer, Berlin/Heidelberg, 2007, pp. 103-112. ISBN: 978-3-540-73280-8.

[2] D.J. Cook, J.C. Augusto, and V.R. Jakkula, Ambient intelligence: Technologies, applications, and opportunities, Pervasive and Mobile Computing, 2009, pp. 277-298.

[3] Y. Ping, M.X. Wu, H. Yu, and G.Q. Xiao, The challenges for the adoption of m-health, in: Service Operations and Logistics, and Informatics, SOLI 'O6, IEEE International Conference, 2006, pp. 181-186, 21-23. 